\title{
Further Discussions on Induced Bias Matrix Model for the Pair-wise Comparison Matrix
}

\author{
Daji Ergu $^{1,2}$ Gang Kou $^{1} \quad$ Yi Peng ${ }^{1, *} \quad$ János Fülöp $^{3} \quad$ Yong Shi $^{4,5}$
}

\begin{abstract}
Inconsistency issue of pairwise comparison matrices has been an important subject in the analytical network process. The most inconsistent elements can efficiently be identified by inducing a bias matrix only based on the original matrix. This paper further discusses the induced bias matrix, and integrates all related theorems and corollaries into the induced bias matrix mode. The theorem of inconsistency identification is proved mathematically using the maximum eigenvalue method and the contradiction method. In addition, a fast inconsistency identification method for one pair of inconsistent elements is proposed and proved mathematically. Two examples are used to illustrate the proposed fast identification method. The results show that the proposed new method is easier and faster than the existing method for the special case with only one pair of inconsistent elements in the original comparison matrix.
\end{abstract}

AMS Classification Number: 47N10; 65K10.

\footnotetext{
This research has been partially supported by grants from the National Natural Science Foundation of China (\#70901011 and \#71173028 for Yi Peng, \#70901015 for Gang Kou, and \#70921061 for Yong Shi), the Fundamental Research Funds for the Central Universities and Program for New Century Excellent Talents in University (NCET-10-0293).

${ }^{1}$ School of Management and Economics, University of Electronic Science and Technology of China, Chengdu, 610200, China.

${ }^{2}$ Southwest University for Nationalities, Chengdu, 610054, China.

${ }^{3}$ Research Group of Operations Research and Decision Systems, Computer and Automation Research Institute, Hungarian Academy of Sciences, H-1111 Kende u. 13-17, Budapest, Hungary.

${ }^{4}$ College of Information Science \& Technology, University of Nebraska at Omaha, Omaha, NE 68182, USA.

${ }^{5}$ Research Center on Fictitious Economy and Data Sciences, Chinese Academy of Sciences, Beijing 100190, China.
} 
Keywords : Analytic network process (ANP); the induced bias matrix model (IBMM); Inconsistency identification; Reciprocal pairwise comparison matrix (RPCM)

\section{Introduction}

The pair-wise comparison method is a well-established technique, and widely used in multi-criteria decision making $(\mathrm{MCDM})$ methods $[1,2]$. The consistency test of pair-wise comparison matrix in the AHP and ANP, two of the widely used MCDM methods, has been studied extensively over the past few decades [3-14]. To improve the consistency ratio of the pair-wise comparison matrix in the AHP and ANP and preserve the original comparison information as much as possible, literature [15] introduced an induced bias matrix (IBM, hereinafter), which can be derived by the original reciprocal pairwise comparison matrix (RPCM hereinafter), to identify and adjust the inconsistent elements.

IBM is based on the theorems of matrix multiplication and vectors dot product as well as the definitions and notations of the pair-wise comparison matrix. The IBM method has been applied in questionnaire design [16], risk analysis [17], and task scheduling [18]. If the comparison matrix $\mathrm{A}$ is perfectly consistent, we mathematically proved that the IBM should be a zero matrix in [15]. If the comparison matrix A is approximately consistent, we also mathematically proved that the IBM should be as close as possible to a zero matrix in [19]. This corollary can be used to estimate the uncertain or missing values in an RPCM. If the pair-wise matrix A is inconsistent, there must be some inconsistent elements in the induced bias matrix (IBM) deviating far away from zero (Corollary 2 in [15]). This corollary shows that 
the farthest value should be identified as the most inconsistent element from the induced bias matrix (IBM). However, this critical corollary for identifying the most inconsistent element has not been proved mathematically in [15].

The objective of this paper is to prove the theorem of aforementioned critical corollary mathematically, and integrates all related theorems and corollaries into the induced bias matrix model (IBMM), which simplifies and refines the proposed inconsistency identification method. In addition, this paper proposes a fast inconsistency identification method to extend the proposed IBMM for the special case of one pair of inconsistent elements in the original RPCM.

The remaining parts of this paper are organized as follows. The next section integrates all related theorem and corollaries into one model and provides two mathematic proofs of Corollary 2.2 (i.e. Theorem 2.3 in IBMM) by maximum eigenvalue method and contradiction method. Section 3 analyzes the inconsistency identification method; proposes and proves a fast inconsistency identification method; describes the sign of non-zero of the induced bias matrix; and introduces two numeric examples with one pair of inconsistent elements to demonstrate the proposed fast inconsistency identification method. Section 4 concludes the paper.

\section{Theorems and Proofs of the Induced Bias Matrix Model (IBMM)}

In order to efficiently identify the inconsistent elements and preserve most of the original pair-wise comparison information, we proposed an induced bias matrix (IBM), which is only based on the original RPCM in [15], and the following theorem and corollaries were derived. 
Theorem 2.1: The induced bias matrix (IBM) $C=A A-n A$ should be a zero matrix, if comparison matrix $A$ is perfectly consistent.

Corollary 2.1: The induced bias matrix (IBM) $C=A A-n A$ should be as close as possible to zero matrix, if comparison matrix $A$ is approximately consistent.

Corollary 2.2: There must be some inconsistent elements in induced bias matrix (IBM) $C$ deviating far away from zero, if the pair-wise matrix A is inconsistent.

The correctnesses of Theorem 2.1 and Corollary 2.1 have been proved mathematically in Section 3.1 of [15] and in Section 2.1 of [19], respectively. Besides, some special cases, where there are some errors in the original RPCM of order 3 , have been addressed as examples in [19]. To determine whether a comparison pairwise matrix is reciprocal, Corollary 2.3 is proposed and illustrated using $3 \times 3$ comparison pair-wise matrix, as an example in [19].

Corollary 2.3: Despite that the comparison matrix $A$ is consistent or not, all entries in the main diagonal of the induced bias matrix (IBM) $C=A A-n A$ should be zeroes, giving that the comparison matrix $A$ is satisfied with the reciprocal condition.

The inconsistent elements are identified by inducing a bias matrix $\mathrm{C}$ from the original RPCM, and the critical component of the above mentioned theorem and corollaries is the induced bias matrix (IBM). To simplify and refine the proposed inconsistency identification method, and make the proposed method more comprehensive and systematic, we integrate these theorem and corollaries into one 
model, say induced bias matrix model (IBMM), which includes the following three theorems.

\section{The Theorem of the Induced Bias Matrix Model (IBMM):}

Theorem 2.2: The induced bias matrix (IBM) $C=A A-n A$ should be equal (or close) to a zero matrix, if comparison matrix $A$ is perfectly (or approximately) consistent. That is,

$$
C=A A-n A \begin{cases}=0 & \text { if } a_{i k} a_{k j}=a_{i j}, \\ \approx 0 & \text { if } a_{i k} a_{k j} \approx a_{i j},\end{cases}
$$

where $A$ is the original RPCM and $a_{i j}$ represents the values of RPCM. The " $n$ " denotes the order of RPCM.

Theorem 2.3: There must be some inconsistent elements in the induced bias matrix (IBM) $C$ deviating far away from zero, if the pair-wise matrix is inconsistent. Especially, any row or column of matrix C contains at least one positive element.

Theorem 2.4: All entries in the main diagonal of the induced bias matrix (IBM) $C=A A-n A$ should be zeroes whether matrix $A$ is consistent or not, as long as the comparison matrix $A$ satisfies the reciprocal condition.

We provided the proof of Theorem 2.2 for consistent case in [15]. The principles of Theorem 2.3 and Theorem 2.4 are demonstrated by introducing some errors into a $3 \times 3 \mathrm{RPCM}$ in [19]. The following subsections mathematically prove Theorem 2.3 and Theorem 2.4.

\subsection{The Proof of Theorem 2.3 by Maximum Eigenvalue Method}

Proof: If the RPCM A is inconsistent, the induced bias matrix $C=A A-n A$ 
cannot be zero. More precisely, any row of C contains at least one positive element.

It is known, e.g. literature [20], that for the maximal eigenvalue $\lambda_{\max }$ of $\mathrm{A}$, $\lambda_{\max } \geq n$, and the corresponding unique eigenvector $\omega_{\max }$ is a positive vector. Furthermore, $\mathrm{A}$ is consistent if and only if $\lambda_{\max }=n$. By applying

$$
A \omega_{\mathrm{max}}=\lambda_{\mathrm{ma}} \omega_{\mathrm{ma}}
$$

at the appropriate places, we get

$$
\begin{aligned}
C \omega_{\max } & =(A A-n A) \omega_{\max }=\lambda_{\max } A \omega_{\max }-n \lambda_{\max } \omega_{\max } \\
& =\lambda_{\max }^{2} \omega_{\max }-n \lambda_{\max } \omega_{\max }=\lambda_{\max }\left(\lambda_{\max }-n\right) \omega_{\max } .
\end{aligned}
$$

Since $\lambda_{\max }>n, C \omega_{\max }$ is a positive vector. Consequently, $C$ cannot have any row containing only zeros. Moreover, since both $C \omega_{\max }$ and $\omega_{\max }$ are positive vectors, any row of $C$ must contain at least one positive element.

\subsection{The Proof of Theorem 2.3 by Contradiction}

Proof: It has been proved in [15] that, if a reciprocal pairwise comparison matrix (RPCM) is perfectly consistent, that is, $a_{i j}=a_{i k} a_{k j}$ for all $i, j, k$, then

$$
c_{i j}=\sum_{k=1}^{n} a_{i k} \cdot a_{k j}-n a_{i j}=n a_{i j}-n a_{i j}=0 .
$$

If an RPCM A is inconsistent, $a_{i j} \neq a_{i k} a_{k j}$ holds at least for one of the $i, j, k$ $(i, j, k=1,2, \cdots, n)$. Moreover, if $A$ is inconsistent, for any $i$, there exist $j$ and $k$ such that $a_{i j} \neq a_{i k} a_{k j}$ (Corollary 2 in [21]). Assume that an RPCM $A$ is inconsistent, but the $i$-th row of the induced bias matrix $\mathrm{C}$ contains only non-positive elements. Then $a_{i j} \neq a_{i k} a_{k j}$ with some $j$ and $k$, and $c_{i 1} \leq 0, c_{i 2} \leq 0, \cdots, c_{i n} \leq 0$. We get the following inequalities: 


$$
\begin{gathered}
\left\{\begin{array}{c}
c_{i 1}=\sum_{k=1}^{n} a_{i k} a_{k 1}-n a_{i 1} \leq 0 \\
c_{i 2}=\sum_{k=1}^{n} a_{i k} a_{k 2}-n a_{i 2} \leq 0 \\
\vdots \\
c_{i n}=\sum_{k=1}^{n} a_{i k} a_{k n}-n a_{i n} \leq 0,
\end{array}\right. \\
\Rightarrow\left\{\begin{array}{c}
\frac{1}{a_{i 1}} \sum_{k=1}^{n} a_{i k} a_{k 1} \leq n \\
\frac{1}{a_{i 2}} \sum_{k=1}^{n} a_{i k} a_{k 2} \leq n \\
\vdots \\
\frac{1}{a_{i n}} \sum_{k=1}^{n} a_{i k} a_{k n} \leq n .
\end{array}\right.
\end{gathered}
$$

Adding all the inequalities together in the system of inequalities (6), we get

$$
\begin{aligned}
& \frac{1}{a_{i 1}} \sum_{k=1}^{n} a_{i k} a_{k 1}+\frac{1}{a_{i 2}} \sum_{k=1}^{n} a_{i k} a_{k 2}+\cdots+\frac{1}{a_{i n}} \sum_{k=1}^{n} a_{i k} a_{k n} \leq n^{2}, \\
& \Rightarrow \sum_{k=1}^{n} \frac{1}{a_{i 1}} a_{i k} a_{k 1}+\sum_{k=1}^{n} \frac{1}{a_{i 2}} a_{i k} a_{k 2}+\cdots+\sum_{k=1}^{n} \frac{1}{a_{i n}} a_{i k} a_{k n} \leq n^{2}, \\
& \Rightarrow \sum_{j=1}^{n} \sum_{k=1}^{n} \frac{1}{a_{i j}} a_{i k} a_{k j}=\sum_{j=1}^{n} \sum_{k=1}^{n} a_{k j} \frac{a_{i k}}{a_{i j}} \leq n^{2} .
\end{aligned}
$$

The inequality (8) or (9) can be unfolded to the following matrix form:

$$
\begin{gathered}
\cdots \frac{1}{a_{i 1}} a_{i 1} a_{11}+\frac{1}{a_{i 1}} a_{i 2} a_{21}+\frac{1}{a_{i 1}} a_{i 3} a_{31}+\cdots+\frac{1}{a_{i 1}} a_{i i} a_{i 1}+\cdots+\frac{1}{a_{i 1}} a_{i j} a_{j 1}+\cdots+\frac{1}{a_{i 1}} a_{i n} a_{n 1}+ \\
\frac{1}{a_{i 2}} a_{i 1} a_{12}+\frac{1}{a_{i 2}} a_{i 2} a_{22}+\frac{1}{a_{i 2}} a_{i 3} a_{32}+\cdots+\frac{1}{a_{i 2}} a_{i i} a_{i 2}+\cdots+\frac{1}{a_{i 2}} a_{i j} a_{j 2}+\cdots+\frac{1}{a_{i 2}} a_{i n} a_{n 2}+ \\
\frac{1}{a_{i 3}} a_{i 1} a_{13}+\frac{1}{a_{i 3}} a_{i 2} a_{23}+\frac{1}{a_{i 3}} a_{i 3} a_{33}+\cdots+\frac{1}{a_{i 3}} a_{i i} a_{i 3}+\cdots+\frac{1}{a_{i 3}} a_{i j} a_{j 3}+\cdots+\frac{1}{a_{i 3}} a_{i n} a_{n 3}+ \\
\vdots \\
\vdots \\
\frac{1}{a_{i i}} a_{i 1} a_{1 i}+\frac{1}{a_{i i}} a_{i 2} a_{2 i}+\frac{1}{a_{i i}} a_{i 3} a_{3 i}+\cdots+\frac{1}{a_{i i}} a_{i i} a_{i i}+\cdots+\frac{1}{a_{i i}} a_{i j} a_{j i}+\cdots+\frac{1}{a_{i i}} a_{i n} a_{n i}+ \\
\vdots \\
\vdots \\
\frac{1}{a_{i j}} a_{i 1} a_{1 j}+\frac{1}{a_{i j}} a_{i 2} a_{2 j}+\frac{1}{a_{i j}} a_{i 3} a_{3 j}+\cdots+\frac{1}{a_{i j}} a_{i i} a_{i j}+\cdots+\frac{1}{a_{i j}} a_{i j} a_{i j}+\cdots+\frac{1}{a_{i j}} a_{i n} a_{n j}+ \\
\vdots \\
\vdots \\
\frac{1}{a_{i n}} a_{i 1} a_{1 n}+\frac{1}{a_{i n}} a_{i 2} a_{2 n}+\frac{1}{a_{i n}} a_{i 3} a_{3 n}+\cdots+\frac{1}{a_{i n}} a_{i i} a_{i n}+\cdots+\frac{1}{a_{i n}} a_{i j} a_{j n}+\cdots+\frac{1}{a_{i n}} a_{i n} a_{n n} \leq n^{2} .
\end{gathered}
$$


Since matrix A is a reciprocal matrix, that is, $a_{k j}=\frac{1}{a_{j k}}$ and $a_{k j}>0, a_{i j}>0$, $a_{i k}>0$, from the expansion inequality (9), any of the above inequalities (7)-(10) can be simplified as the following inequality:

$$
\begin{array}{r}
n+\sum_{k>j}\left(a_{k j} \frac{a_{i k}}{a_{i j}}+a_{j k} \frac{a_{i j}}{a_{i k}}\right) \leq n^{2}, \\
\Rightarrow \sum_{k>j}\left(a_{k j} \frac{a_{i k}}{a_{i j}}+a_{j k} \frac{a_{i j}}{a_{i k}}\right) \leq n^{2}-n .
\end{array}
$$

Since there are $\frac{n(n-1)}{2}$ sum term at the left side of the inequality (12), and $a_{k j} \frac{a_{i k}}{a_{i j}}+a_{j k} \frac{a_{i j}}{a_{i k}}=a_{k j} \frac{a_{i k}}{a_{i j}}+\frac{1}{a_{k j} \frac{a_{i k}}{a_{i j}}} \geq 2$, the inequality (12) holds if and only if $a_{k j} \frac{a_{i k}}{a_{i j}}=1$, namely, $a_{i j}=a_{i k} a_{j k}$ for all $j$ and $k$. However, this result contradicts the previous assumption that $a_{i j} \neq a_{i k} a_{k j}$ for some $j$ and $k$. Therefore, one of the inequalities, at least, does not hold. Thus, inequality (12) holds with > sign. This entails that at least one of elements in the $i$-th row the induced bias matrix $\mathrm{C}$ is positive.

Based on the above two proofs for rows, the same proofs for columns can also be induced. If $\mathrm{A}$ is a pairwise comparison matrix with the reciprocal property, the transpose of $\mathrm{A}$ is also a pairwise comparison matrix with the reciprocal property. In addition, $\mathrm{A}$ is consistent if and only if the transpose of $\mathrm{A}$ is consistent.

The transpose of the IBM $\mathrm{C}$ generated by $\mathrm{A}$ is the IBM generated by the transpose of $\mathrm{A}$. Consequently, if $\mathrm{C}$ is inconsistent, any column of $\mathrm{C}$ contains at least one positive element. The same statement for the rows was stated earlier 


\subsection{The Proof of Theorem 2.4}

Proof: According to the principle of matrix multiplication, all values in the main diagonal of the induced bias matrix $\mathrm{C}$ can be calculated by the formula (13):

$$
c_{i i}=\sum_{k=1}^{n} a_{i k} \cdot a_{k i}-n a_{i i} .
$$

If $a_{i k}=\frac{1}{a_{i k}}$, and $a_{i i}=1$, then

$$
c_{i i}=\sum_{k=1}^{n} a_{i k} \cdot a_{k i}-n a_{i i}=\sum_{k=1}^{n} a_{i k} \cdot \frac{1}{a_{i k}}-n a_{i i}=n-n=0, \quad i=1,2, \cdots, n .
$$

\section{The Inconsistency Identification Method}

In this section, the mathematic principles of the proposed "Method of Maximum", "Method of Minimum" and "Method for identifying $a_{i j}$ " in [15], are firstly discussed. Next, a fast inconsistency identification method for special case is proposed and proved mathematically. Two numerical examples are introduced to illustrate the proposed method in Section 3.3. Details are given next.

\subsection{Clarification of the Proposed Inconsistency Identification Method}

If the RPCM A is inconsistent, the above proof shows that any row of the IBM C contains at least one non-zero element, that is, $a_{i j} \neq a_{i k} a_{k j}$ holds at least for one group of $i, j, k$, which means that there is at least one pair of inconsistent elements existing in the original RPCM A. Suppose $c_{i j}$, the element with largest absolute value in the IBM C, is identified. The second step is to analyze that which element makes $c_{i j}$ to be far away from zero. According to the rule of matrix multiplication, the value 
of $c_{i j}$ is calculated by all values on the $i^{\text {th }}$ row and $j^{\text {th }}$ column of matrix A and $a_{i j}$, that is,

$$
\begin{aligned}
& c_{i j}=\sum_{k=1}^{n} a_{i k} \cdot a_{k j}-n a_{i j} \\
& =a_{i 1} a_{1 j}-a_{i j}+a_{i 2} a_{2 j}-a_{i j}+\cdots+a_{i k} a_{k j}-a_{i j}+\cdots+a_{i n} a_{n j}-a_{i j} .
\end{aligned}
$$

Clearly, the farthest value of $c_{i j}$ can be impacted by any term of $a_{i k} a_{k j}-a_{i j}$ on the right side of the sum equality (15). In order to identify the inconsistent elements that caused the value of $c_{i j}$ to be far away from zero, the scalar product of vectors in $n$ dimension technique is introduced. The impact of each term can easily be observed by the scalar product of vectors in $n$ dimension technique, that is,

$$
b=r_{i} \cdot c_{j}^{T}=\left(a_{i 1}, a_{i 2}, \cdots, a_{i n}\right) \cdot\left(a_{1 j}, a_{2 j}, \cdots, a_{n j}\right)=\left(a_{i 1} a_{1 j}, a_{i 2} a_{2 j}, \cdots, a_{i n} a_{n j}\right),
$$

and

$$
f=b-a_{i j}=\left(a_{i 1} a_{1 j}-a_{i j}, a_{i 2} a_{2 j}-a_{i j}, \cdots a_{i k} a_{k j}-a_{i j}, \cdots, a_{i n} a_{n j}-a_{i j}\right) .
$$

If $a_{i j} \neq a_{i k} a_{k j}$, then $a_{i k} a_{k j}-a_{i j} \neq 0$. Therefore, the non-zero element(s), which caused the value of $c_{i j}$ to be far away from zero, can be identified through observing all elements in the bias identifying vector $f$. In addition, the inequality $a_{i j} \neq a_{i k} a_{k j}$ can be caused by $a_{i j}$ or any $a_{i k} a_{k j}(k=1,2, \cdots, n)$, or both.

Obviously, if the inconsistent element is $a_{i j}$, other elements are consistent. Assume $a_{i k} a_{k j}=a_{i j}^{\prime}>a_{i j}$, namely, $a_{i j}$ is too small. We can get that all values in the bias identifying vector $f$ are positive except $k=i, j$, as $a_{i i} a_{i j}-a_{i j}=0$ and $a_{i j} a_{j j}-a_{i j}=0$. Vice versa, if $a_{i j}$ is too large, all the values in the bias identifying vector $f$ will be negative except two values $k=i, j$. Therefore, the "Method for identifying $a_{i j}$ " inconsistency identification method is proposed [15]. 
Besides, the farthest value of $c_{i j}$ must be caused by some outliers either too large or too small located at the bias identifying vector $f$; therefore, "Method for Maximum" and "Method for Minimum" inconsistency identification methods are proposed [15].

In order to further identify the inconsistent element for those elements whose values are close to the largest or smallest simultaneously, therefore the "Method of matrix order reduction" inconsistency identification method is proposed [15].

\subsection{Fast Identification Method for Special Case and Its Proof}

In this section, one fast inconsistency identification method is proposed to quickly identify the inconsistent elements when there is only one pair of inconsistent elements in the original RPCM.

Assume that RPCM A is inconsistent, and there is one pair of inconsistent elements $a_{i p}$ and its corresponding reciprocal element $a_{p i}=\frac{1}{a_{i p}}$, while other elements are consistent, namely, $a_{i k} a_{k j}=a_{i j}$ for all $k$ except $k=p\left(a_{i p} a_{p j} \neq a_{i j}\right)$. Therefore, the two inconsistent elements are elements at the $i^{\text {th }}$ and $p^{\text {th }}$ rows, and the $p^{\text {th }}$ and $i^{\text {th }}$ columns. According to the rule of matrix multiplication, all elements, which are located at the $i^{\text {th }}, p^{\text {th }}$ rows, and the $i^{\text {th }}, p^{\text {th }}$ columns in the induced bias matrix $C=A A-n A$, will be impacted by $a_{i p}$ and $a_{p i}$. Since it is assumed that $a_{i k} a_{k j}=a_{i j}(k \neq p ; j \neq p$,$) and a_{i k} a_{k p} \neq a_{i p}$, suppose $a_{i k} a_{k p}=a_{i p}^{\prime}$, all the values in the $i^{\text {th }}$ row of the IBM C can be computed by formula (18), that is,

$$
c_{i j}=\sum_{k=1}^{n} a_{i k} \cdot a_{k j}-n a_{i j}=\sum_{k=1, \neq p}^{n} a_{i k} \cdot a_{k j}+a_{i p} a_{p j}-n a_{i j}, \quad j=1,2, \cdots, n
$$




$$
=\left\{\begin{array}{l}
(n-1) a_{i j}+a_{i p} a_{p j}-n a_{i j}=a_{i p} a_{p j}-a_{i j} ; \quad j \neq i, p \\
(n-1)+1-n=0 ; \quad j=i \\
(n-2) a_{i p}^{\prime}+a_{i i} a_{i p}+a_{i p} a_{p p}-n a_{i p}=(n-2)\left(a_{i p}^{\prime}-a_{i p}\right) ; j=p .
\end{array}\right.
$$

In order to analyze the sign change of each element on the $i^{\text {th }}$ row, the equalities in (18) are further unfolded, as shown below.

$$
\left\{\begin{aligned}
c_{i 1}= & a_{i p} a_{p 1}-a_{i 1} \\
c_{i 2}= & a_{i p} a_{p 2}-a_{i 2} \\
& \vdots \\
c_{i i}= & 0 \\
& \vdots \\
c_{i p}= & (n-1) a_{i p}^{\prime}+a_{i i} a_{i p}+a_{i p} a_{p p}-n a_{i p}=(n-2)\left(a_{i p}^{\prime}-a_{i p}\right) \\
& \vdots \\
c_{i n}= & a_{i p} a_{p n}-a_{i n} .
\end{aligned}\right.
$$

If $a_{i p} \uparrow$, then

$$
\left\{\begin{aligned}
c_{i 1}= & a_{i p} a_{p 1}-a_{i 1}>0 \\
c_{i 2}= & a_{i p} a_{p 2}-a_{i 2}>0 \\
& \vdots \\
c_{i i}= & 0 \\
& \vdots \\
c_{i p}= & (n-1) a_{i p}^{\prime}+a_{i i} a_{i p}+a_{i p} a_{p p}-n a_{i p}=(n-2)\left(a_{i p}^{\prime}-a_{i p}\right)<0 \\
& \vdots \\
c_{i n}= & a_{i p} a_{p n}-a_{i n}>0 .
\end{aligned}\right.
$$

If $a_{i p} \downarrow$, then

$$
\left\{\begin{aligned}
c_{i 1}= & a_{i p} a_{p 1}-a_{i 1}<0 \\
c_{i 2}= & a_{i p} a_{p 2}-a_{i 2}<0 \\
& \vdots \\
c_{i i}= & 0 \\
& \vdots \\
c_{i p}= & (n-1) a_{i p}^{\prime}+a_{i i} a_{i p}+a_{i p} a_{p p}-n a_{i p}=(n-2)\left(a_{i p}^{\prime}-a_{i p}\right)>0 \\
& \vdots \\
c_{i n}= & a_{i p} a_{p n}-a_{i n}<0,
\end{aligned}\right.
$$

where the symbols " $\uparrow$ ” and " $\downarrow$ ” denote "increase" and "decrease”, respectively 
(hereinafter).

Likewise, for the $p^{\text {th }}$ row:

$$
\begin{aligned}
& c_{p j}=\sum_{k=1}^{n} a_{p k} \cdot a_{k j}-n a_{p j}=\sum_{k=1, \neq i}^{n} a_{p k} \cdot a_{k j}+a_{p i} a_{i j}-n a_{p j}, \quad j=1,2, \cdots, n \\
& =\left\{\begin{array}{l}
(n-1) a_{p j}+a_{p i} a_{i j}-n a_{p j}=a_{p i} a_{i j}-a_{p j} ; \quad j \neq i, p \\
0 ; \\
(n-1) a_{p j}^{\prime}+a_{p i} a_{i i}+a_{p p} a_{p i}-n a_{p i}=(n-2)\left(a_{p i}^{\prime}-a_{p i}\right) ; j=i .
\end{array}\right.
\end{aligned}
$$

Therefore, if $a_{i p} \uparrow$, then $a_{p i}=\frac{1}{a_{i p}} \downarrow$,

$$
c_{p j}=\left\{\begin{array}{l}
(n-1) a_{p j}+a_{p i} a_{i j}-n a_{p j}=a_{p i} a_{i j}-a_{p j}<0 ; \quad j \neq i, p \\
0 ; \quad j=p \\
(n-1) a_{p j}^{\prime}+a_{p i} a_{i i}+a_{p p} a_{p i}-n a_{p i}=(n-2)\left(a_{p i}^{\prime}-a_{p i}\right)>0 ; \quad j=i .
\end{array}\right.
$$

Likewise, if $a_{i p} \downarrow$, then $a_{p i}=\frac{1}{a_{i p}} \uparrow$,

$$
c_{p j}=\left\{\begin{array}{l}
(n-1) a_{p j}+a_{p i} a_{i j}-n a_{p j}=a_{p i} a_{i j}-a_{p j}>0 ; \quad j \neq i, p \\
0 ; \quad j=p \\
(n-1) a_{p j}^{\prime}+a_{p i} a_{i i}+a_{p p} a_{p i}-n a_{p i}=(n-2)\left(a_{p i}^{\prime}-a_{p i}\right)<0 ; \quad j=i .
\end{array}\right.
$$

If $a_{i p} \uparrow$, all values on the $i^{\text {th }}$ row of IBM $\mathrm{C}$ will be more than zeroes $\left(c_{i j}>0, j=1,2, \cdots, n\right.$ and $\left.j \neq p\right)$ except $c_{i p}<0$, and all values on the $p^{\text {th }}$ row of IBM C will be less than zeroes $\left(c_{i j}<0, j=1,2, \cdots, n\right.$ and $\left.j \neq p\right)$ except $c_{i p}>0$. Therefore, only the elements on the $i^{\text {th }}$ row and $p^{\text {th }}$ row are non-zeroes, and the sign form of the values on the $i^{\text {th }}$ row and $p^{\text {th }}$ row of the IBM C can be derived, as shown in the following matrix, 


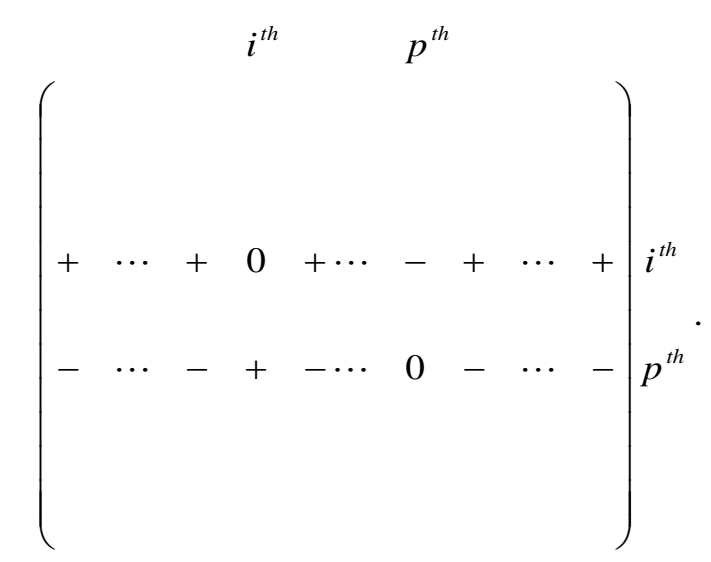

Likewise, the signs of each element on the $i^{\text {th }}$ column and $p^{\text {th }}$ column can be derived similarly.

$$
\begin{aligned}
& c_{j i}=\sum_{k=1}^{n} a_{j k} \cdot a_{k i}-n a_{j i}=\sum_{k=1, \neq p}^{n} a_{j k} \cdot a_{k i}+a_{j p} a_{p i}-n a_{j i}, \quad j=1,2, \cdots, n \\
& =\left\{\begin{array}{l}
(n-1) a_{j i}+a_{j p} a_{p i}-n a_{j i}=a_{j p} a_{p i}-a_{j i} ; j \neq p, i \\
0 ; \\
(n-1) a_{p i}^{\prime}+a_{p i} a_{i i}+a_{p p} a_{p i}-n a_{p i}=(n-2)\left(a_{p i}^{\prime}-a_{p i}\right) ; j=p .
\end{array}\right.
\end{aligned}
$$

If $a_{i p} \uparrow$, then $a_{p i}=\frac{1}{a_{i p}} \downarrow$,

$$
c_{j i}=\left\{\begin{array}{l}
(n-1) a_{j i}+a_{j p} a_{p i}-n a_{j i}=a_{j p} a_{p i}-a_{j i}<0 ; \quad j \neq p, i \\
0 ; \quad j=i \\
(n-1) a_{p i}^{\prime}+a_{p i} a_{i i}+a_{p p} a_{p i}-n a_{p i}=(n-2)\left(a_{p i}^{\prime}-a_{p i}\right)>0 ; \quad j=p .
\end{array}\right.
$$

For the $p^{\text {th }}$ column,

$$
\begin{aligned}
& c_{j p}=\sum_{k=1}^{n} a_{j k} \cdot a_{k p}-n a_{j p}=\sum_{k=1, \neq i}^{n} a_{j k} \cdot a_{k p}+a_{j i} a_{i p}-n a_{j p}, \quad j=1,2, \cdots, n \\
& =\left\{\begin{array}{l}
(n-1) a_{j p}+a_{j i} a_{i p}-n a_{j p}=a_{j i} a_{i p}-a_{j p} ; \quad j \neq p, i \\
0 ; \quad j=p \\
(n-1) a_{i p}^{\prime}+a_{i i} a_{i p}+a_{i p} a_{p p}-n a_{i p}=(n-2)\left(a_{i p}^{\prime}-a_{i p}\right) ; \quad j=i,
\end{array}\right.
\end{aligned}
$$

If $a_{i p} \uparrow$,

$$
c_{j p}=\left\{\begin{array}{l}
(n-1) a_{j p}+a_{j i} a_{i p}-n a_{j p}=a_{j i} a_{i p}-a_{j p}>0 ; \quad j \neq p, i \\
0 ; \quad j=p \\
(n-1) a_{i p}^{\prime}+a_{i i} a_{i p}+a_{i p} a_{p p}-n a_{i p}=(n-2)\left(a_{i p}^{\prime}-a_{i p}\right)<0 ; \quad j=i .
\end{array}\right.
$$

Therefore, the sign forms of the elements on the $i^{\text {th }}$ and $p^{\text {th }}$ columns of the 
IBM C can be obtained, as shown in (30)

$$
\left(\begin{array}{ccc}
i^{t h} & p^{t h} \\
- & & + \\
\vdots & & \vdots \\
- & \cdots & + \\
0 & & - \\
- & & + \\
+ & \cdots & 0 \\
- & & + \\
\vdots & & \vdots \\
- & & +
\end{array}\right) i^{\text {th }}
$$

To sum up, if $a_{i p} \uparrow$, the signs of all the values, which are located at the $i^{\text {th }}$ row and the $i^{\text {th }}$ column, the $p^{\text {th }}$ row and $p^{\text {th }}$ column, become:



Therefore, we can obtain the following fast inconsistency identification method:

\section{Method of non-zero rows (columns) and signs identification:}

If there are two rows (the $i^{\text {th }}$ row and $p^{\text {th }}$ row) and two columns (the $i^{\text {th }}$ column and $p^{\text {th }}$ column) with non-zeroes, and other elements are zero, the inconsistent elements must be $a_{i p}$ and $a_{p i}$.

If the $c_{i p}<0$ and other elements located at the $i^{\text {th }}$ row or $p^{\text {th }}$ column are more than zeroes, $a_{i p}$ is too large and should be decreased. Vice versa, $a_{i p}$ is too small and should be increased. 
If the $c_{p i}>0$ and other elements located at the $p^{\text {th }}$ row or $i^{\text {th }}$ column are less than zeroes, $a_{i p}$ is too large and should be decreased. Vice versus, $a_{i p}$ is too small and should be increased.

In addition,

$$
c_{i p}=\sum_{k=1}^{n} a_{i k} \cdot a_{k p}-n a_{i p}=\sum_{k=1, \neq i, p}^{n} a_{i k} \cdot a_{k p}+a_{i i} a_{i p}+a_{p p} a_{i p}-n a_{i p} .
$$

To make the RPCM A be consistent, the value of $c_{i p}$ should be equal to zero. Therefore, inconsistent element $a_{i p}$ can be adjusted by formula (33).

$$
a_{i p}=\frac{1}{n-2} \sum_{k=1, \neq i, p}^{n} a_{i k} \cdot a_{k p}
$$

In this case, since there is only one pair of inconsistent elements, $a_{i p}$ and $a_{p i}$ in the RPCM, any $a_{i k} a_{k p}=a_{i p}^{\prime} \quad(k \neq i, p$,$) can be used as the revised value of a_{i p}$.

\subsection{Illustrative Examples for Fast Inconsistency Identification}

\section{Method}

The Example 2 and Example 3 in [15] are used in this study as Example 3.1 and Example 3.2, respectively, to demonstrate the proposed fast inconsistency identification method.

Example 3.1: The induced bias matrix C, computed by the proposed IBM method in the Example 2 in [15], is

$$
C=\left(\begin{array}{cccc}
0 & -1.9688 & -3.9375 & 15.7500 \\
31.5000 & 0 & 0 & -3.9375 \\
15.7500 & 0 & 0 & -1.9688 \\
-15.7500 & 15.7500 & 31.5000 & 0
\end{array}\right)
$$

It can easily be observed that there are only two non-zero rows (the $1^{\text {st }}$ and $4^{\text {th }}$ ), and two non-zero columns (the $1^{\text {st }}$ and $4^{\text {th }}$ ) in the IBM C, which is identical to the 
formula (31). In addition, there is only one sign different from those elements whether located at the $1^{\text {st }}$ and $4^{\text {th }}$ rows or columns. Therefore, according to the principle of above proposed fast inconsistency identification method, the inconsistent elements are $a_{14}$ and $a_{41}$. Specifically, $a_{14}$ is too small and should be increased since $c_{14}=15.75>0$. According to the revising formula (33) of $a_{i p}$, we get

$$
a_{14}=\frac{1}{4-2} \sum_{k=2}^{3} a_{1 k} a_{k 4}=\frac{1}{2}(2 \times 4+4 \times 2)=8, a_{41}=\frac{1}{a_{14}}=\frac{1}{8} .
$$

The identified inconsistent element and its revised result are the same as shown in Example 2 of [15].

Example 3.2 : The induced bias matrix C, computed by the proposed IBMM in the Example 3 of [15], is showed below.

$$
C=\left(\begin{array}{cccccccc}
0 & -1.8750 & 7.5000 & 0 & 0 & 0 & 0 & 0 \\
7.5000 & 0 & -22.5000 & 15.0000 & 3.7500 & 15.0000 & 3.7500 & 15.0000 \\
-1.8750 & 22.5000 & 0 & -3.7500 & -0.9375 & -3.7500 & -0.9375 & -3.7500 \\
0 & -0.9375 & 3.7500 & 0 & 0 & 0 & 0 & 0 \\
0 & -3.7500 & 15.0000 & 0 & 0 & 0 & 0 & 0 \\
0 & -0.9375 & 3.7500 & 0 & 0 & 0 & 0 & 0 \\
0 & -3.7500 & 15.0000 & 0 & 0 & 0 & 0 & 0 \\
0 & -0.9375 & 3.7500 & 0 & 0 & 0 & 0 & 0
\end{array}\right) .
$$

It can easily be observed that there are only two non-zero rows (the $2^{\text {nd }}$ and $3^{\text {rd }}$ ), and two non-zero columns (the $2^{\text {nd }}$ and $3^{\text {rd }}$ ) in above IBM C. Likewise, there is only one sign different from those elements whether located at the $2^{\text {nd }}$ and $3^{\text {rd }}$ rows or columns. Both are identical to the formula (31). Therefore, according to the above fast identification method, the inconsistent elements are $a_{23}$ and $a_{32}$. Besides, since $c_{23}=-22.5<0$ and $c_{32}=22.5>0$, we can get that $a_{23}$ is too large, and $a_{32}$ is too small. 
Since $c_{23}=\sum_{k=1}^{8} a_{2 k} a_{k 3}-8 a_{23}=\sum_{k=1, \neq 2,3}^{8} a_{2 k} a_{k 3}+a_{22} a_{23}+a_{23} a_{33}-8 a_{23}$, assume $c_{23}=0$, $a_{23}=\frac{1}{6} \sum_{k=1, \neq 2,3}^{8} a_{2 k} a_{k 3}=\frac{1}{6}\left(\frac{1}{2} \times \frac{1}{2}+1 \times \frac{1}{4}+\frac{1}{4} \times 1+1 \times \frac{1}{4}+\frac{1}{4} \times 1+1 \times \frac{1}{4}\right)=\frac{1}{4}$, and $a_{32}=4$. For simplicity, since $a_{23}$ is inconsistent, assume $a_{2 k} a_{k 3}=a_{23}^{\prime},(k=1,4,5,6,7,8)$; we can use $a_{23}^{\prime}$ to be the value of $a_{23}$ in order to let it be consistent. Clearly, any value of pair of $a_{2 k} a_{k 3}=a_{23}^{\prime}(k=1,4,5,6,7,8)$ is $\frac{1}{4}$, which is the same as shown in Example 3 of [15].

The above examples show that the inconsistent elements can be determined by observing and analyzing the non-zero row (column), and sign identification of the bias elements in the IBM C instead of following the seven steps of inconsistency identification developed in [15]. Therefore, the proposed method is simpler and faster than the previous one for the special case with only one pair of inconsistent elements in the original RPCM.

\section{Conclusion}

This paper discussed the principle of the proposed inconsistency identification method. A fast inconsistency identification method for the special case with only one pair of inconsistent elements in the original RPCM was also proposed and proved mathematically. Two examples indicate that the inconsistent elements can be easily and quickly identified by the proposed fast inconsistency identification method if there are only two rows and corresponding two columns with non-zero elements.

Although the special case with only one pair of inconsistent elements in the original RPCM can easily and quickly be identified by the proposed fast inconsistency 
identification method, such cases where there are more than one pair of inconsistent elements in RPCM will be relatively complicated by the proposed method, and it remains to be studied in future.

\section{References}

1. Sun, L., Greenberg, B.: Multicriteria Group Decision Making: Optimal Priority Synthesis from Pairwise Comparisons. Journal of Optimization Theory and Applications, 130(2), pp. 317-339 (2006).

2. Lipovetsky, S.: Global Priority Estimation in Multiperson Decision Making. Journal of Optimization Theory and Applications, 140(1), pp.77-91 (2009).

3. Saaty, T.L.: Axiomatic Foundation of the Analytic Hierarchy Process, Management Science, 32 (7), 841-855 (1986).

4. Blankmeyer, E.: Approaches to consistency adjustment. Journal of Optimization Theory and Applications, 54(3), pp. 479-488 (1987).

5. Saaty, R.W.: The analytic hierarchy process-what it is and how it is used, Mathematical Modelling, 9 (3-5), 161-176 (1987).

6. Harker, P. \& Vargas, L.: The theory of ratio scale estimation: Saaty's analytic hierarchy process. Management Science 33 (11), 1383-1403 (1987).

7. Saaty, T.L.: How to make a decision: The analytic hierarchy process, European Journal of Operational Research, 48 (1), 9-26 (1990).

8. Xu, Z. \& Wei, C.: A consistency improving method in the analytic hierarchy process, European Journal of Operational Research, 116, 443-449 (1999). 
9. Li, H. \& Ma L.: Detecting and adjusting ordinal and cardinal inconsistencies through a graphical and optimal approach in AHP models. Computers \& Operations Research 34 (3), 780-798 (2007).

10. Cao, D., Leung, L.C. \& Law, J.S.: Modifying inconsistent comparison matrix in analytic hierarchy process: A heuristic approach. Decision Support Systems, 44, 944-953 (2008).

11. Iida, Y.: Ordinality Consistency Test About Items And Notation Of A Pairwise Comparison Matrix in AHP. Proceedings of the International Symposium on the Analytic Hierarchy Process. http://www.isahp.org/2009Proceedings/Final_Papers/ 32_Iida_Youichi_ConsistencyTest_in_Japan_REV_FIN.pdf (2009).

12. Koczkodaj, W. W \& Szarek, S. J.: On distance-based inconsistency reduction algorithms for pairwise comparisons. Logic Journal of the IGPL, 18 (6), 859-869 $(2010)$

13. Peng, Y., Kou, G., Wang, G., Wu, W., and Shi, Y.: Ensemble of software defect predictors: an AHP-based evaluation method, International Journal of Information Technology \& Decision Making, 10(1), 187-206 (2011).

14. Kou, G., Lu, Y., Peng, Y., and Shi, Y.: Evaluation of Classification Algorithms using MCDM and Rank Correlation, International Journal of Information Technology \& Decision Making, 11(1), 197-225 (2012). DOI: $10.1142 / \mathrm{S} 0219622012500095$. 
15. Daji Ergu, Gang Kou, Yi Peng, Yong Shi: A Simple Method to Improve the Consistency Ratio of the Pair-wise Comparison Matrix in ANP, European Journal of Operational Research, 213(1) 246-259 (2011). DOI: 10.1016/j.ejor.2011.03.014.

16. Daji Ergu, Gang Kou: Questionnaire Design Improvement and Missing Item Scores Estimation for Rapid and Efficient Decision Making, Annals of Operations Research, DOI: 10.1007/s10479-011-0922-3 (2011).

17. Daji Ergu, Gang Kou, Yong Shi, Yu Shi: Analytic Network Process in Risk Assessment and Decision Analysis, Computers \& Operations Research, DOI: 10.1016/j.cor.2011.03.005, 2011.

18. Daji Ergu, Gang Kou, Yi Peng, Yong Shi, Yu Shi: The Analytic Hierarchy Process: Task Scheduling and Resource Allocation in Cloud Computing Environment, The Journal of Supercomputing. DOI: 10.1007/s11227-011-0625-1, 2011.

19. Daji Ergu, Gang Kou, Yi Peng, Yong Shi, Yu Shi: BIMM: A Bias Induced Matrix Model for Incomplete Reciprocal Pairwise Comparison Matrix, Journal of Multi-Criteria Decision Analysis, 18(1) 101-113 (2011). DOI: 10.1002/mcda.472.

20. Saaty, T.L.: The Analytical Hierarchy Process. McGraw-Hill, New York(1980).

21. Bozóki, S., Fülöp, J. \& Poesz, A.: On pairwise comparison matrices that can be made consistent by the modification of a few elements. Central European Journal of Operations Research, 19, 157-175 (2011). 